\title{
Characterization of Rosemary Essential Oil for biodegradable emulsions
}

\author{
S. Rodríguez-Rojo*, S. Varona, M. Núñez, M. J. Cocero \\ Universidad de Valladolid. Escuela de Ingenierías Industriales - Sede Dr. Mergelina- \\ c/ Doctor Mergelina s/n 47011 Valladolid, España \\ * Corresponding author: sorayarr@iq.uva.es
}

\section{Abstract}

2 The characterization of rosemary essential oil (EO) for its formulation in biodegradable

3 emulsions has been carried out. Firstly, the required HLB (hydrophile-lipophile balance) value

4 of the oil was determined to be 15 based on droplet size analysis and the stability of emulsions

5 with synthetic surfactants. Moreover the emulsion resulted to be stable after 50 days of

6 storage in ambient conditions. Secondly, four biodegradable and non-toxic surfactants derived

7 from starch were tested. The effect of these surfactants was analyzed by measuring interfacial

8 tension between the oil and the aqueous phase.

9 Keywords: Essential oil, emulsion, required HLB, surface tension, surfactant

\section{1. Introduction}

11 Essential oils (EOs) are commonly used as natural preservatives and fragrances in cosmetic

12 products. More recently, thanks mainly to their antimicrobial properties, new applications as

13 food preservatives (Burt, 2004), growth promoters in livestock (Calsamiglia et al., 2007),

14 natural pesticides in organic agriculture (El-Shafei et al., 2010) and insecticides (Phillips et al.,

15 2010) are emerging. EOs typically are volatile and they rapidly evaporate from surfaces. It is thus desirable to formulate them, in its effective concentration, in a way that allows minimizing the evaporation and protecting the oil, from high temperature, oxidation and UV light, at the same time. Besides, such formulations should allow for a selective release and for the increase of the shelf life of the oil. For this purpose, oil-in-water (O/W) emulsions are one of the preferred formulations of EOs. 

HLB (hydrophile-lipophile balance) values close to that required for the oil phase (Aulton, 1995). The required HLB values of commonly used fixed and mineral oils have been determined but those of EOs are yet to be studied.

The HLB of rosemary EO has been determined according to the method proposed and validated by Orafidiya and Oladimeji (2002) based on the determination of droplet size and size distribution of emulsions prepared with surfactants of a wide range of HLB values, being the required HLB for the essential oil that of the surfactant leading to the most stable emulsion, i.e. that of the minimum droplet size.

As previously mentioned, this required HLB value of the oil phase will be useful to select an appropriate surfactant for the emulsion. These data is mainly available for synthetic surfactants or it can be calculated from the chemical formula by group contribution methods (Tadros, 2005; Wang et al., 2011). However, this information is not always available for natural or natural derived surfactants, such as n-octenyl succinic anhydride (OSA)-modified starches, whose application in emulsion formulation is increasing in the last years (Varona et al., 2009; Wang et al., 2011). In such cases, the measurement of the interfacial tension between the oil and a water solution of the surfactant whose HLB value is unknown, provide reliable and fast information for the selection of the appropriate surfactant and the minimum concentration needed to obtain a stable emulsion (Huang et al., 2011) which is generally higher than the critical micelle concentration (CMC) for the corresponding surfactant, provided that this information is available. The smaller the interfacial tension between the oil phase and water, the more stable will be the emulsion as it adsorbs onto the oil forming interfacial film, reducing the interactions between both phases and the energy required to expand the interface of the phase to be dispersed, in this case the oily one, for the formation of the emulsion (Tadros, 2005). 
Hence, in the second part of this work the interfacial tension between the Rosemary EO and

47 the water for four biodegradable and non-toxic surfactants, n-octenyl succinic anhydride

48 (OSA)- modified starches, was measured and compared with the interfacial tension of the EO and a synthetic surfactant with a value of HLB equal to that required HLB by the EO, in order to select the most adequate OSA-starch and the minimum concentration required to obtain a stable emulsion.

\section{Materials and Methods}

\subsection{Materials}

54 The essential rosemary (Rosmarinus officinalis L.) oil was supplied by COCOPE (Peñafiel, Spain). It was obtained by steam distillation. The surfactants, $\operatorname{Span}^{\circledR} 20$, Tween $^{\circledR} 20$ and Tween $^{\circledR} 80$, were purchased from Sigma (Spain). The (OSA)-modified starches were kindly provided by National Starch Food Innovation (Hamburg, Germany). The followings were tested: OSA-starch derived from waxy maize (OSA-1), OSA-starch derived from waxy maize blend with dried glucose syrup (OSA-2), OSA-dextrin derived from waxy maize (OSA-3) and OSA-dextrin derived from tapioca (OSA-4).

\subsection{Methods}

\subsubsection{Preparation of the emulsion}

Oil-in-water emulsions were prepared by a two-step process. A surfactant solution ( $200 \mathrm{~mL})$ was initially prepared by dissolving the surfactant at a concentration of $1 \mathrm{~g} / \mathrm{L}$ in deionized water

67 (Milli-Q, Millipore) with the aid of a magnetic stirrer (IKA). Afterwards $50 \mathrm{~mL}$ essential oil was gradually added to the suspension under continuous agitation for 5 min and a crude emulsion was obtained. The resulting coarsely dispersed raw emulsion was then fed into the rotor-stator 
machine (IKA ${ }^{\circledR}$ LABOR PILOT 2000/4) and processed during 2 min for fine emulsification at

$7170 \mathrm{~Hz}$, according to Varona et al, 2009.

\subsubsection{Preparation of the mixture of surfactants}

Emulsions were prepared using a combination of two surfactants, Span 20 whose HLB is 8.6 and Tween 20 whose HLB is 16.7. The proportion between these two surfactants was changed in order to obtain HLB values in-between. The HLB of the mixture was calculated according to:

$H L B=x_{A} \cdot H L B_{A}+x_{B} \cdot H L B_{B}(1)$

where $\mathrm{x}_{\mathrm{A}}$ and $\mathrm{x}_{\mathrm{B}}$ are the weight fractions of each surfactant.

\subsubsection{Droplet size analysis}

Rosemary oil droplet size distribution of the emulsion was determined by laser diffraction

(Mastersizer 2000, Malvern Instruments) with a dual-wavelength detection system (Red light: $633 \mathrm{~nm}$ and Blue light: $436 \mathrm{~nm}$ ). The sample was diluted with deionized water in the dispersion unit (Hydro SM) to less than $1 \%(\mathrm{v} / \mathrm{v})$ to prevent multiple scattering effects. The refractive index of the dispersed phase, rosemary essential oil, was experimentally determined (Stanley Abbey Refractometer) to be 1.468 , in good agreement with literature values (Atti-Santos et al., 2005). Particle size measurements are reported as the $10 \%, 50 \%$ and $90 \%$ quantiles of the volume distribution, i.e. the $50 \%$ volume of droplets have a size smaller than the $d_{50, v}$ value;

87 The values are the average of 3 measurements. The maximum incertitude of the analysis was $5 \%$, except for stored emulsions of HLB below 10.7 that reached values up to $20 \%$. In order to observe variation during the storage time, the measurements were made directly

90 after the production of the emulsion, and 21 and 50 days later following the same procedure.

\subsubsection{Determination of the stability of emulsions}

92 The emulsion stability, that means the ability of an emulsion to resist changes in its properties with time, was analyzed using two different criteria: the amount of de-emulsified oil at two 
94

95

96

97

time intervals ( 21 and 50 days) and the kinetics of sedimentation and creaming of the emulsion in the following 60 minutes after preparation.

For both analysis, $7 \mathrm{~mL}$ of the emulsion were poured in a vertical glass tube with an inner diameter of $13 \mathrm{~mm}$ (height of emulsion: $55 \mathrm{~mm}$ ) and stored at ambient conditions.

The height of visible supernatant oil layer was recorded, and compared to maximum height of oil $(11 \mathrm{~mm})$, i.e. the emulsion were completely de-emulsified; this ratio multiple by 100 was the \% of de-emulsionated oil.

Particles migration phenomena (creaming or sedimentation) induce particle volume fraction changes at the extremities of the sample. The creaming and sedimentation rates were followed using a Turbiscan Classic MA2000 (Formulaction, France). The stability of emulsions is followed measuring the variation of \%backscattering of an $860 \mathrm{~nm}$ near infrared beam versus the height of the sample, in steps of $40 \mu \mathrm{m}$, every $2 \mathrm{~min}$ for $60 \mathrm{~min}$. The backscattering detector receives the light scattered backward by the sample at an angle of $45^{\circ}$ with respect to the light source. The software of the equipment determines the calculation the slopes of the \%backscattering curves versus time in the lower and upper part of the emulsion (Figure 2, inner box) in order to evaluate the kinetics of sedimentation and creaming, respectively. These rates are then used to calculate the thickness of sedimentation and creaming after a defined time (i.e. 60 minutes) for each emulsion in order to compare them.

\subsubsection{Evaluation of modified starch surfactants}

In order to evaluate the performance of the modified starch surfactant, the interfacial tension between the EO and the aqueous phase was measured. The concentration of surfactant in the aqueous phase ranged from 0 to $100 \mathrm{~g} / \mathrm{L}$. The interfacial tension was compared with that achieved with a synthetic surfactant with a HLB equal to that required by the rosemary EO. Measurements were made in triplicate using the Wilhelmy plate method with a tensiometer 

measurements was $2 \mathrm{~min}$. The maximum standard deviation was $5 \%$.

\section{Experimental results and discussion}

\subsection{Required HLB}

122 The required HLB value for the rosemary essential oil is between 12 and 15 . According to the 123 analysis of mean drop size (Figure 1), expressed as the $50 \%$ quantile $\left(\mathrm{d}_{50, v}\right)$, a defined HLB cannot be identified for a clear minimum value of the droplet size. The de-emulsified oil after 50 days was neither a clear criteria, as only emulsions prepared with a surfactant mixture HLB value smaller than 10.7 show a measurable ( $>1 \mathrm{~mm}$ ) layer of supernatant oil. Besides, the emulsion prepared with a surfactant of HLB 14 displayed a thin layer of oil with a height lower than $1 \mathrm{~mm}$. Only, the emulsions prepared with surfactants of HLB 8.6 and 9.2 were completely de-emulsified after 50 days.

The analysis of the backscattered light across the emulsion allows determining the height of emulsion sedimentation and creaming (e) for each emulsion, to evaluate its stability in the first hour after their preparation (Figure 2). In a similar way to previous criteria of stability, all the emulsions prepared with mixtures of surfactants with a HLB value higher than 10.7 have similar values of " $\mathrm{e}$ ". In order to point out a specific value of required HLB in the range from 12 to 15 , the width of the droplet size distribution (DSD) based on the $10 \%$ and $90 \%$ quantile was analyzed. As

137 shown in Figure 3, fresh emulsions prepared with surfactant mixtures with HLB between 10.7

138 and 16.7 present a very narrow DSD. As the emulsions evolved with time, the width of the DSD increases; after 21 days the initial HLB range is reduced to 13 to 15 , and after 50 days, the narrowest DSD is the one of the emulsion prepared with a surfactant mixture of HLB equal to 15. Hence, it can be concluded that the required HLB of the rosemary essential oil is 15 . 
This value is slightly higher than the values of other essential oils for aromatic herbs from the

143 order of Lamiales, such as lavandin oil with a value of 13.4 (Varona et al., 2009), lippia and

144 peppermint oils with values of 12.1 and 12.3, respectively (Orafidiya and Oladimeji, 2002).

145 Although, the HLB may be related with the hydrophilicity of the main component of the

146 essential oil, this is not always the case due to the great amount of components present in the

147 oil. In the lavandin oil, the main component is the linalool (ca. 45\%), which is more hydrophilic

148 than the menthone (Phillips et al., 2010), the main component in the peppermint oil (ca.45\%-

$14960 \%)$. However, the main component of the rosemary oil used in this work is the 1,8-cineole

150 (ca. 24\%), which is more hydrophobic than the linalool, although HLB value of rosemary oil is

151 higher than that of lavandin oil. These can be due to the presence of other minor components

152 more hydrophilic, such as camphor (ca. 16\%).

\subsection{Evaluation of modified starch surfactants}

154

As shown in Figure 4, the most adequate modified starches to be employed as surfacts with rosemary essential oil, are OSA-4 and OSA-2, as they deacrease the interfacial tension between water and rosemary EO from $16.2 \pm 0.6 \mathrm{mN} / \mathrm{m}$ to a lower value with respect to the other surfactants tested. As reference, the interfacial values using a synthetic surfactant with the HLB value requiered by the rosemary EO $\left(\operatorname{Tween}^{\circledR} 80, \mathrm{HLB}=15\right)$ are also presented. The minimum concentration of the surfactant that should be used to prepare stable emulsions of rosemary EO is $25 \mathrm{~g} / \mathrm{L}$, which is 5 times higher than the critical micelle concentration of these two surfactants, $4.5 \mathrm{~g} / \mathrm{L}$ and $5.2 \mathrm{~g} / \mathrm{L}$ for OSA-2 and OSA-4, respectively (Varona et al., 2009).

\section{Conclusions}

The characterization of rosemary EO regarding its formulation in emulsion has been carried out. A required HLB value of 15 was determined according to the stability of emulsions in terms of minimum droplet size and width of the drop size distribution over a period of 50 days storage at ambient conditions. The performance of four biodegradable and non-toxic 
surfactants derived from starch as stabilizers for rosemary essential oil emulsions in water, was

tested based on interfacial tension measurements. The best surfactants, OSA-2 and OSA-4,

reduced the interfacial tension between water and the essential oil in two thirds.

\section{Acknowlegdments}

171 The authors thank the financial support of Junta de Castilla y Leon (Spain) through the project GR11/2008. National Starch Group is acknowledged for supplying the OSAmodified starches and giving permission to publish the results obtained with them. S.Rodríguez-Rojo thanks the Spanish Ministry of Education for her postdoctoral grant. S. Varona thanks the University of Valladolid through the FPI-UVa research program.

\section{References}

Atti-Santos, A. C., Rossato, M., Pauletti, G.F., Rota, L. D., Rech, J.C., Pansera, M.R., Agostini, F., Serafini, L. A., Moyna, P., 2005. Physico-chemical Evaluation of Rosmarinus officinalis L. Essential Oils. Braz. arch. biol. technol. 48, 1035-1039

Aulton, M.E. (Ed.), 1995. Pharmaceutics: The Science of Dosage Form Design. Churchill Livingstone, New York, pp. 290-292. a review. Int. J. Food Microbiol. 94, 223- 253. modifiers of rumen microbial fermentation. J. Dairy Sci. 90, 2580-2595.

187 friendly pesticides: Essential oil-based w/o/w multiple emulsions for anti-fungal formulations. Ind. Crop Prod. 31, 99-106. 
191 Orafidiya, L., Oladimeji, F.A., 2002. Determination of the required HLB values of some essential 192 oils. Int. J. Pharm. 237, 241-249.

193 Phillips, A. K., Appel, A. G., Sims, S. R., 2010. Topical Toxicity of Essential Oils to the German

194 Cockroach (Dictyoptera: Blattellidae), J. Econ. Entomol. 103, 448-459.

195 Tadros, T.F., 2005. Applied Surfactants: Principles and Applications. Wiley-VCH Verlag GmbH \& 196 Co.KGaA. Weinheim (Germany).

197 Varona. S, Martín. A, Cocero. M.J., 2009. Formulation of a natural biocide based on lavandin 198 essential oil by emulsification using modified starches. Chem. Eng. Process. 48, 1121-1128.

199 Wang, X., Li, X., Chen, L., Fengwei, X., Yu, L., Li, B., 2011. Preparation and characterisation of 200 octenyl succinate starch as a delivery carrier for bioactive food components. Food Chem. 126, 201 $1218-1225$ 
Tables

Table 1. Main components of the rosemary essential oil analyzed by GC

\begin{tabular}{|l|r|}
\hline Component & wt. $\%$ \\
\hline 1,8-cineole & 23.8 \\
\hline$\alpha$-pinene & 15.6 \\
\hline camphor & 15.6 \\
\hline camphene & 9.2 \\
\hline Bornyl acetate & 5.7 \\
\hline$\beta$-pinene & 5.1 \\
\hline Borneol & 4.4 \\
\hline caryophillene & 3.9 \\
\hline$\alpha$-terpineol & 1.5 \\
\hline
\end{tabular}


Figures and captions

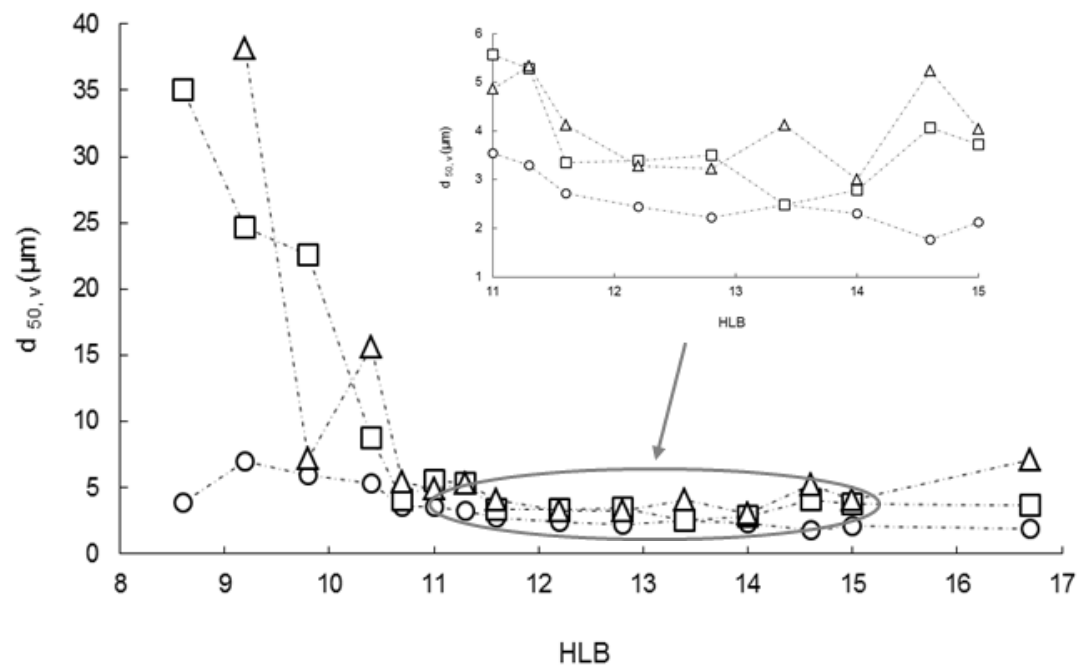

Figure 1. Drop emulsion diameter for different HLB values. Evolution with time: 0 - 0 days (preparation), $\square-21$ days and $\Delta-50$ days. 


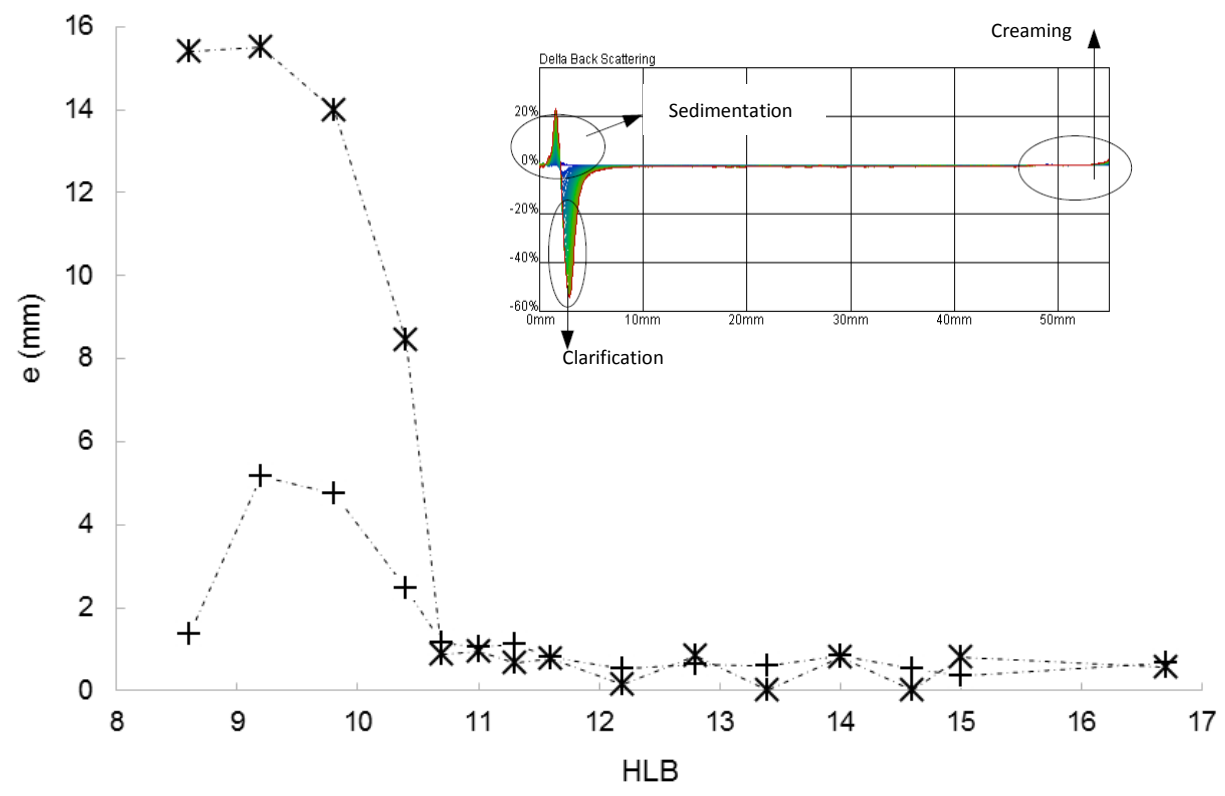

Figure 2. Thickness of the layer of sedimentation $(+)$ and creaming $(*)$ phenomena in the emulsion 60 minutes after preparation for different HLB values of the surfactant. Inner box: Backscattering profiles of the emulsion prepared with a HLB mixture value of 10.7, taking as reference the backscattering scan at time 0 . Source data for the determination of the thickness of the referred layers. 


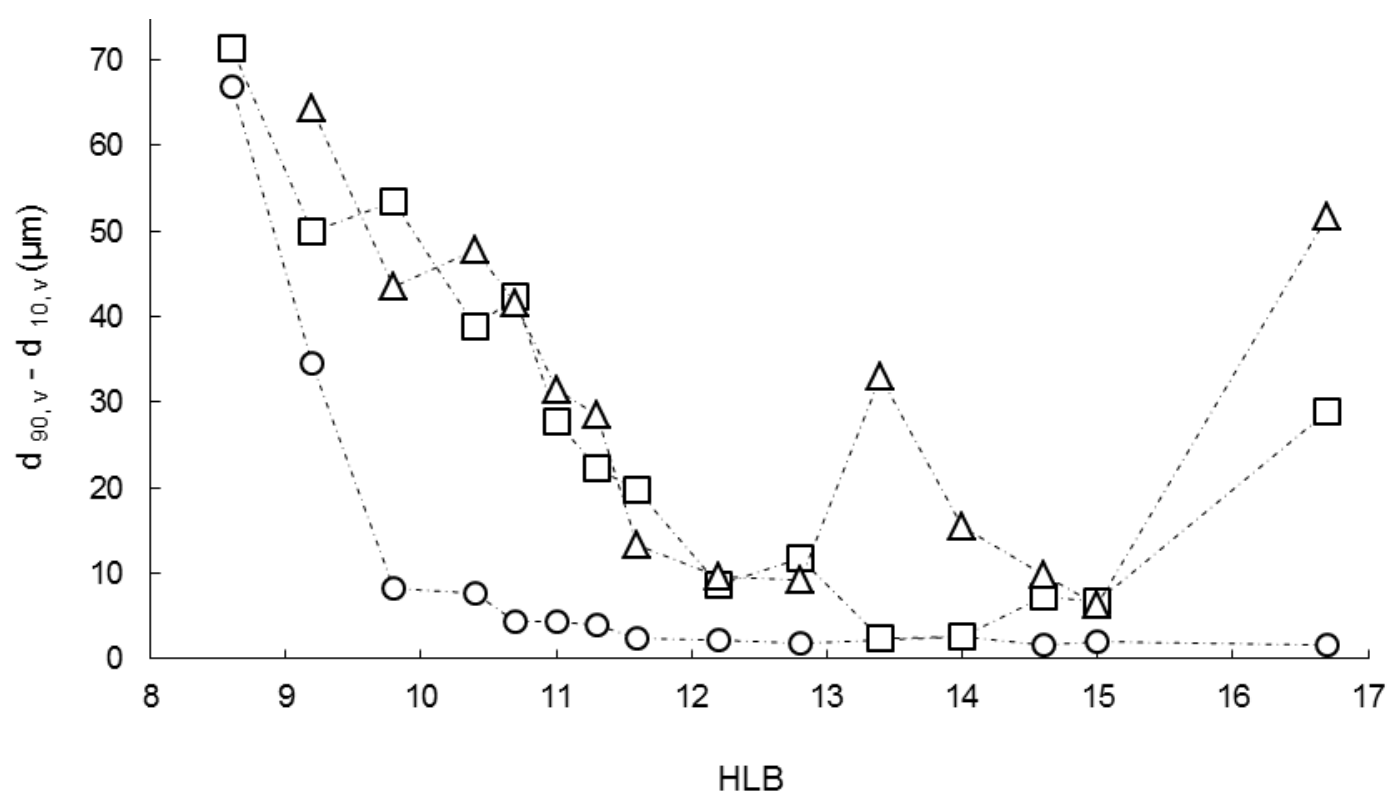

Figure 3. Width of particle size distribution $\left(d_{90, v}-d_{10, v}\right)$ for different HLB values. Evolution with time: $\bigcirc-0$ days (preparation), $\square-21$ days and $\Delta-50$ days. 


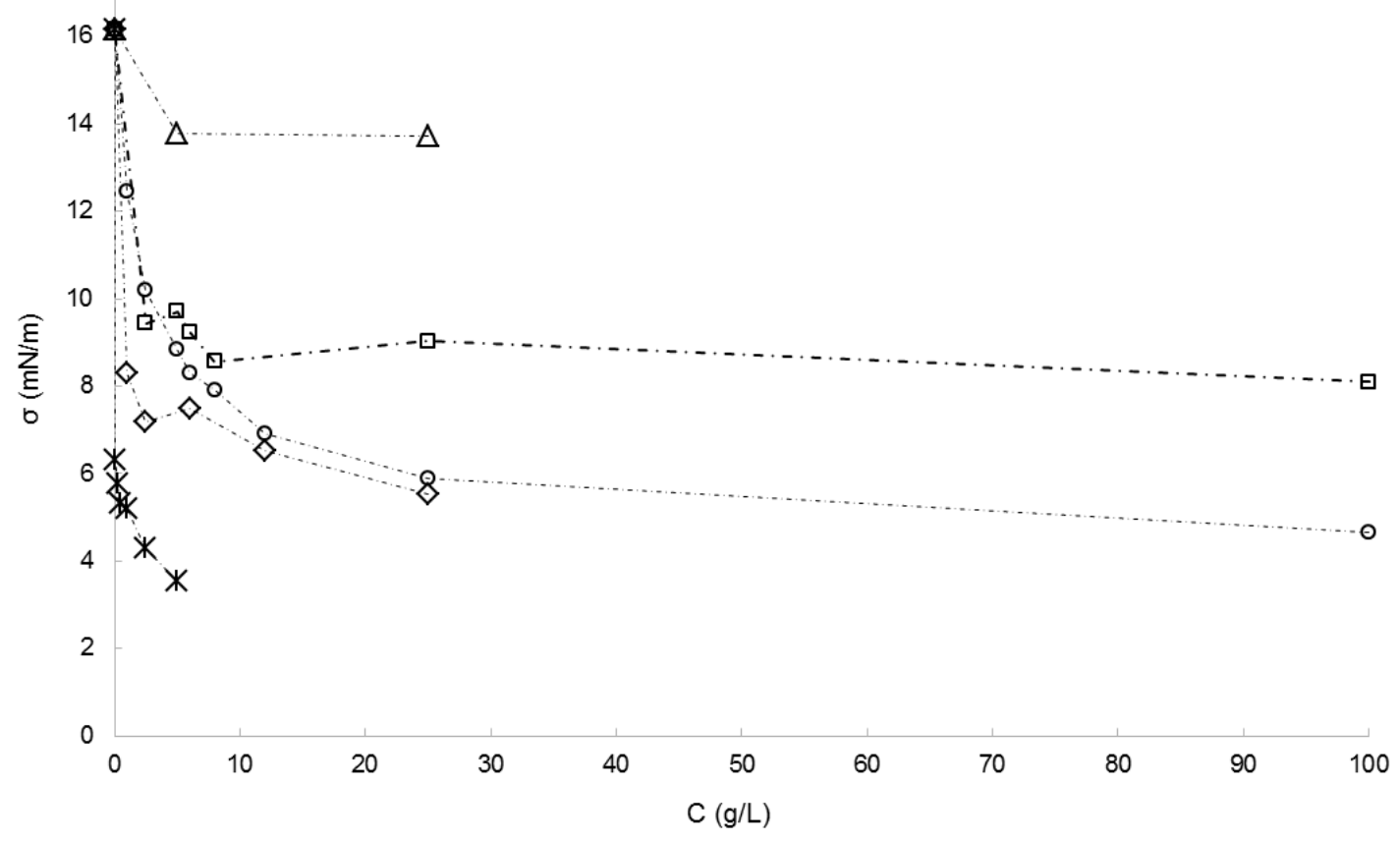

Figure 4. Reduction in interfacial tension water/ rosemary EO with the addition of surfactants:

OSA-1 ( $\square)$, OSA-2 $(\diamond)$, OSA-3 $(\Delta)$, OSA-4 (O) and Tween $80(*)$. 